Metastasiertes Nierenzellkarzinom

\title{
Die Erstlinientherapie ist entscheidend
}

Derzeit stehen verschiedene zielgerichtete Therapien für die Erstlinienbehandlung des metastasierten Nierenzellkarzinoms (mRCC) zur Verfügung. Wichtig ist eine möglichst effektive Therapie von Beginn an.

$\mathrm{Zu}$ den Substanzen der Erstlinientherapie zählen der Multityrosinkinase-Inhibitor Sunitinib $\left(\right.$ Sutent ${ }^{\circledR}$ ) und der mTOR-Inhibitor Temsirolimus (Torisel ${ }^{\circledR}$ ). „Die Entscheidung, welches Medikament in der Erstlinientherapie zum Einsatz kommt, richtet sich maßgeblich nach der Effektivität.
Was wir am Anfang nicht erreicht haben, können wir später nicht mehr einholen“, unterstrich Michael Staehler, München. Eine geringere Tumorlast bedeute für viele Patienten eine bessere Lebensqualität. Mit einer objektiven Ansprechrate von $47 \%$ und einem medianen Gesamtüberleben von 26,4 Monaten ist Sunitinib Referenzstandard in der Erstlinientherapie des metastasierten Nierenzellkarzinoms. Die hohen Ansprechraten und die günstigen Überlebensdaten sind der Grund dafür, dass die interdisziplinäre Konsensuskonferenz mRCC Su- nitinib als Erstlinientherapie bei Patienten mit guter und mittlerer Prognose empfohlen hat.

Für mRCC-Hochrisikopatienten ist Temsirolimus Erstlinienstandard und die einzige zielgerichtete Substanz, die bei dieser Patientengruppe einen signifikanten Überlebensvorteil zeigen konnte. Die Substanz ist dabei zur Behandlung fortgeschrittener Nierenzellkarzinome von Patienten zugelassen, die mindestens drei von sechs prognostisch ungünstigen Faktoren aufweisen. $\mathrm{Zu}$ diesen Risikofaktoren zählen z. B. mehr als zwei Organe mit Metastasen, hohes LDH, hohes Kalzium oder niedriges Hämoglobin. AF

Nach Informationen von Pfizer Pharma

Kurz notiert

Effektive Erhaltungstherapie für

AML-Patienten

Die zielgerichtete Behandlung der akuten myeloischen Leukämie (AML) konnte in den letzten Jahren deutlich verbessert werden. Bei einem Satellitensymposium anlässlich des Deutschen Krebskongresses 2012 in Berlin bezeichneten die Experten eine Erhaltungstherapie mit Histamindihydrochlorid, HDC (Ceplene ${ }^{\circledast}$ ) und Interleukin-2 als wichtige Therapieoption für die AML-Patienten. Sie präsentierten eine aktuelle Phase-III-Studie, in der diese immuntherapeutisch wirksame Kombination das leukämiefreie Überleben der Studienteilnehmer signifikant verlängern konnte. Bei AML-Patienten in erster kompletter Remission mit nachweisbarer Resterkrankung, die nicht für eine allogene Stammzelltransplantation infrage kommen, konnte so das Rezidivrisiko vermindert und die Remission stabil erhalten werden.

Nach Informationen von MEDA Pharma

Neue Image-Broschüre von Roche Aktuell ist die Broschüre „Roche Personalisierte Medizin. Kleine Unterschiede, große Wirkung" erschienen. Darin erklärt das Unternehmen, wie es das Prinzip der personalisierten Medizin bei verschiedenen Indikationen
- beispielsweise bei Krebserkrankungen im Bereich von Magen, Brust und Lunge - umsetzt. Besonders hervorgehoben wird das jüngste Beispiel eines personalisierten Therapieansatzes beim metastasierten Melanom: Die EMA (european medicines agency) hat im Februar 2012 Vemurafenib (Zelboraf ${ }^{\mathrm{TM}}$ ) für die Behandlung des inoperablen oder metastasierenden Melanoms zugelassen, das eine BRAFV600-Mutation aufweist. Vemurafenib hemmt gezielt mutierte Formen des BRAF-Proteins, die bei rund der Hälfte aller Fälle des malignen Melanoms festgestellt werden.

Nach Informationen von Roche

\section{Kolonkarzinom: Bewährte Targeting Therapy}

Mit der randomisierten Phase-III-Studie PRIME wurde bei 1.183 Patienten mit metastasiertem Kolonkarzinom untersucht, welchen Einfluss

b die Addition von Panitumumab (Vectibix ${ }^{\oplus}$ ) zur Erstlinientherapie mit 5-FU, Folinsäure und Oxaliplatin besitzt. Unter zusätzlicher Gabe von Panitumumab war das progressionsfreie Überleben signifikant länger (9,6 vs. 8,0 Monate), erklärte Michael Stahl, Essen. Stahl betonte zudem, dass auch bei Kolonkarzinomen mit auf die Leber beschränkter Metastasierung Panitumumab effektiv eingesetzt werden könne: In der PRIME-Studie war gegenüber der alleinigen Chemotherapie eine Steigerung der R0-Resektionsraten von 10,4 auf $27,9 \%$ möglich. eb

Nach Informationen von Amgen

Neues aus der Onkologie-Pipeline

Mit dem besseren Verständnis der Tumorbiologie werden auch neuartige zielgerichtete Ansätze in der onkologischen Therapie geprüft. Dazu zählt z. B. Alpharadin (Radium223-Chlorid). Der Alphastrahler soll zur Behandlung von Karzinomen mit Knochenmetastasen eingesetzt werden. Ähnlich wie natürliches Kalzium lagert sich Alpharadin gezielt in Regionen mit erhöhtem Knochenstoffwechsel in und um Knochenmetastasen an. Erste Erfolge konnten beim metastasierten Prostatakarzinom gezeigt werden.

Regorafenib ist ein oral wirksamer MultiKinase-Inhibitor, der in die Neoangiogenese eingreift. Die Substanz konnte bei Patienten mit metastasierten kolorektalen Karzinomen ebenfalls in einer Phase-III-Studie eine Verlängerung des Gesamtüberlebens zeigen. Zu den weiteren Projekten gehört etwa ein bi-spezifischer Antikörper (BiTE ${ }^{\circledast}$ ), der gezielt gegen ein Antigen der Prostata gerichtet ist. eb Nach Informationen von Bayer Vital 